\title{
ERME Topic Conference: Mathematics Teaching, Resources and Teacher Professional Development
}

\section{5-7 October 2016, Humboldt-Universität zu Berlin, Germany}

Stefan Zehetmeier (University of Klagenfurt, Austria), Bettina Rösken-Winter (Humboldt-Universität zu Berlin, Germany, Despina Potari (National and Kapodistrian University of Athens, Greece) and Miguel Ribeiro (Campinas State University (UNICAMP), Campinas, Brazil)

Mathematics teaching and mathematics teacher professional development are areas where research has increased substantially in the last years. For the last ERME conferences, a large number of proposals was related to this research area (e.g., three topic groups were formed at CERME9 in 2015: TWG18 on mathematics teacher education; TWG19 on mathematics teaching; and TWG20 on resources for teaching).

In this ongoing field of research, many issues need further investigation. We need to better understand the underlying characteristics of mathematics teacher education and the professional development contexts that have a positive impact on teachers' professional learning, even with respect to sustainability. Also, further discussion and research are needed on how to link research findings and how to bridge theoretical and methodological approaches to mathematics teacher pre-service and in-service education.

Studying mathematics teaching goes beyond teachers' classroom behavior. It encompasses teachers' actions and meaning-making as these relate to instruction. This includes, amongst others, task selection and design, classroom communication and assessment as well as the interplay between goals and actions as classroom interactions unfold in the context of broader institutional, educational, and social settings. A central question for investigation is what kind of methodological and theoretical tools are necessary to address this complexity.

In terms of resources, the focus of research for the last decades has been on teachers' beliefs and knowledge. More recently, teachers' identity, tasks, and teaching resources have received attention. Moreover, mathematics teacher educators' knowledge and development has been an emerging field. Aiming at achieving a better understanding, characterizing and/or evaluating the content of teachers' knowledge, several theoretical and methodological frameworks have been developed and discussed. Yet, further discussion seems to be needed in order to better describe the content of such knowledge, its relationships with (and influence on) teachers' beliefs, goals and identity as well as with mathematics teaching.

These three strands (mathematics teacher education, teaching and resources) are far from being disconnect- ed. The ERME Topic conference "Mathematics Teaching, Resources and Teacher Professional Development" (5-7 October 2016, Humboldt-Universität zu Berlin, Germany) served as a platform for investigating in what ways these strands are linked - as regards research questions, methodologies and theoretical perspectives. The International Programme Committee was chaired by Stefan Zehetmeier (Austria), Miguel Ribeiro (Brazil), Bettina Rösken-Winter (Germany), and Despina Potari (Greece).

The conference focused on exchanging participants' knowledge and experiences, and on networking between scholars from different countries and cultures. In sum 69 scholars (60 from Europe) from 16 countries (12 from Europe) participated in this conference and submitted 37 papers and 14 posters. All submissions were peer-reviewed and a selection was made according to the quality of the work and the potential to contribute to the conference themes. Finally, 27 papers and 12 posters were accepted to be presented at the conference. Pre-conference proceedings were published online on the conference website (https://www.hu-berlin.de/de/einrichtungen-organisation/ wissenschaftliche-einrichtungen/zentralinstitute/pse/erme/ erme-topic-conference). A selection of extended papers will be published within the ERME book series.

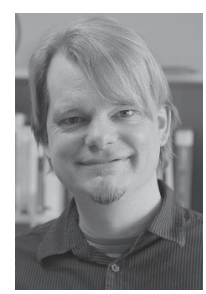

Stefan Zehetmeier is an associate professor at the University of Klagenfurt (Austria). His research interests include teacher education, school development, evaluation and impact analysis of teacher professional development programmes.

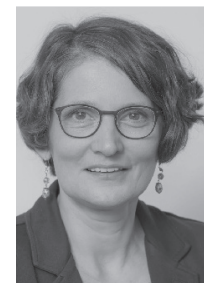

Bettina Rösken-Winter is a professor for Design-Based Research and Mathematics Education at the Humboldt-Universität zu Berlin (Germany). Her main research interests are related to developing and evaluating courses to support and enhance mathematics teachers' continuous professional development. 


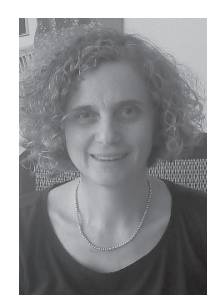

Despina Potari is a professor in mathematics education at the University of Athens (Greece). Her main research interests have been on the development of mathematics teaching and learning and teacher development at different educational levels. Her current research focus is on the researchpractice relation and on the role of different contexts and tools in classroom and PD settings.
Miguel Ribeiro concluded his PhD with a research focusing on teachers' knowledge, beliefs and mathematical goals. His research interests include kindergarten to secondary mathematics teachers specialized knowledge and teacher trainers specialized knowledge. 